\title{
Neutron Inelastic Scattering Studies of Globular Compounds
}

L. N. Becka

Citation: The Journal of Chemical Physics 38, 1685 (1963); doi: 10.1063/1.1776941

View online: https://doi.org/10.1063/1.1776941

View Table of Contents: http://aip.scitation.org/toc/jcp/38/7

Published by the American Institute of Physics

\section{PHYSICS TODAY}

ADVANCED LIGHT CURE ADHESIVES

READ NOW

Take a closer look at what these environmentally friendly adhesive systems can do
PRESENTED BY

(7) MASTERBOND 


\title{
Neutron Inelastic Scattering Studies of Globular Compounds
}

\author{
L. N. BeCKA ${ }^{*} \dagger$ \\ Atomic Energy of Canada Limited, Chalk River, Ontario, Canada
}

(Received 6 December 1962)

\begin{abstract}
The use of neutron inelastic scattering measurements to determine the nature of the solid-state transitions in globular compounds is proposed and exemplified. Patterns were taken of the high- and low-temperature phases of solid cyclohexane, 2-2-dimethylbutane, and 1-4-diazo-bicyclo-(2.2.2)-octane. The first two have broad inelastic scattering peaks in the low-temperature phase. In the high-temperature phase these peaks disappear and the elastic peak broadens markedly. These changes are attributed to "quasi-free-rotation" of the molecules. Inelastic peaks do not appear in 1-4-diazo-bicyclo-(2.2.2)-octane, and the elastic peak does not broaden appreciably in the high-temperature phase. Self-diffusion coefficients $D_{\mathrm{a}}=4.5 \times 10^{-5} \mathrm{~cm}^{2} / \mathrm{sec}$ for cyclohexane and $D_{s}=8.6 \times 10^{-5} \mathrm{~cm}^{2} / \mathrm{sec}$ for 2-2-dimethylbutane were determined from the patterns of the liquids at $300^{\circ} \mathrm{K}$ using the "small motions" approximation. These values are larger than those obtained from NMR measurements, presumably as a result of irreversible rotation of the molecules.
\end{abstract}

M ANY globular compounds ${ }^{1}$ are characterized by relatively high melting points, high vapor pressures, first-order transitions with large entropy changes in the solid state, and a certain degree of plasticity in the high-temperature phase. The phase transitions are from a low-temperature ordered phase, to a highly disordered high-temperature phase. The nature of the transition has been studied by $\mathrm{x}$-ray diffraction, ${ }^{2}$ nuclear magnetic resonance ${ }^{3}$ (hereafter NMR), calorimetry, ${ }^{4}$ and by means of measurements of dielectric constants in polar molecules. ${ }^{5}$ Of these methods x-ray diffraction and calorimetry cannot distinguish clearly between a passage to a dynamically disordered phase (molecules reorienting almost freely) or to a statistically disordered phase (molecules in different but fixed orientations in different cells). The existence of rotations in the solid can be detected by NMR since it will narrow the linewidth and reduce the second moment of the line. Also, measurements of spin-lattice relaxation times give reorientation rates. Polar molecules with freedom to reorient will give high dielectric constants, so that here also we have a method for establishing the existence of rotation in the solid.

In addition to the above mentioned methods, there exists the possibility of detecting the existence of "quasi-free-rotation" in the solid by the use of neutron inelastic-scattering experiments. ${ }^{6}$ If the intermolecular

\footnotetext{
1 J. Timmermans, Phys. Chem. Solids 18, 1 (1961).

* National Research Council (Canada) Post-doctoral Fellow.

$\dagger$ Present address: Departmento de Química Inorgánica y FísicoQuímica, Facultad de Ciencias Exactas y Naturales, Perí 222, Buenos Aires, Argentina.

${ }^{2}$ W. J. Dunning, Phys. Chem. Solids 18, 21 (1961).

${ }^{3}$ E. R. Andrew, Phys. Chem. Solids 18, 9 (1961).

4 K. A. L. Staveley, Phys. Chem. Solids 18, 46 (1961).

${ }^{5}$ C. P. Smyth, Phys. Chem. Solids 18, 40 (1961).

B. N. Brookhouse, Inelastic Scattering of Neutrons in Solid and Liquids (I.A.E.A., Vienna, 1961), p. 113.
}

forces opposing rotation in the solid are very weak and a large proportion of molecules are able to surmount this potential barrier, the neutron-scattering pattern should show a very broad peak, centered near the elastic peak, whose width diminishes markedly with decreasing $Q$ (momentum transfer in units of $\hbar$ ). Furthermore, if there are inelastic scattering peaks due to modes of intermolecular vibrations in the lowtemperature phase, they should disappear above the transition temperature, i.e., they will move to the region of almost zero energy transfer. The work described in this paper was done to demonstrate the foregoing statements, establishing at the same time the nature of the disorder in some globular compounds. Experiments were also made on these substances in the liquid state. Since the intermolecular forces in some of them should be similar to those in the inert gases, presenting experimental advantages over the latter (ease of handling, highly incoherent scattering), it is considered that a study of them should be rewarding in order to elucidate the structural dynamics of liquids. The work described here on liquids is only illustrative.

The substances studied were cyclohexane, 2-2-dimethylbutane and 1-4-diazo-bicyclo-(2.2.2)-octane (hereafter DABCO) in the solid state. The first two were also studied in the liquid state. Cyclohexane has an fcc crystal structure in the high-temperature phase and its $x$-ray diffraction pattern shows evidence of disorder. ${ }^{7}$ There is a first-order phase change at $186^{\circ} \mathrm{K}$ below which the crystal symmetry is perhaps monoclinic. ${ }^{8}$ NMR studies show that there is almost isotropic reorientation of the molecules at a rate of at

${ }^{7}$ T. Oda, X-Sen 5, 26 (1948) (C.A. 44, 5179d).

${ }^{8}$ G. S. Krishna Murti, Indian J. Phys. 32, 460 (1958). 


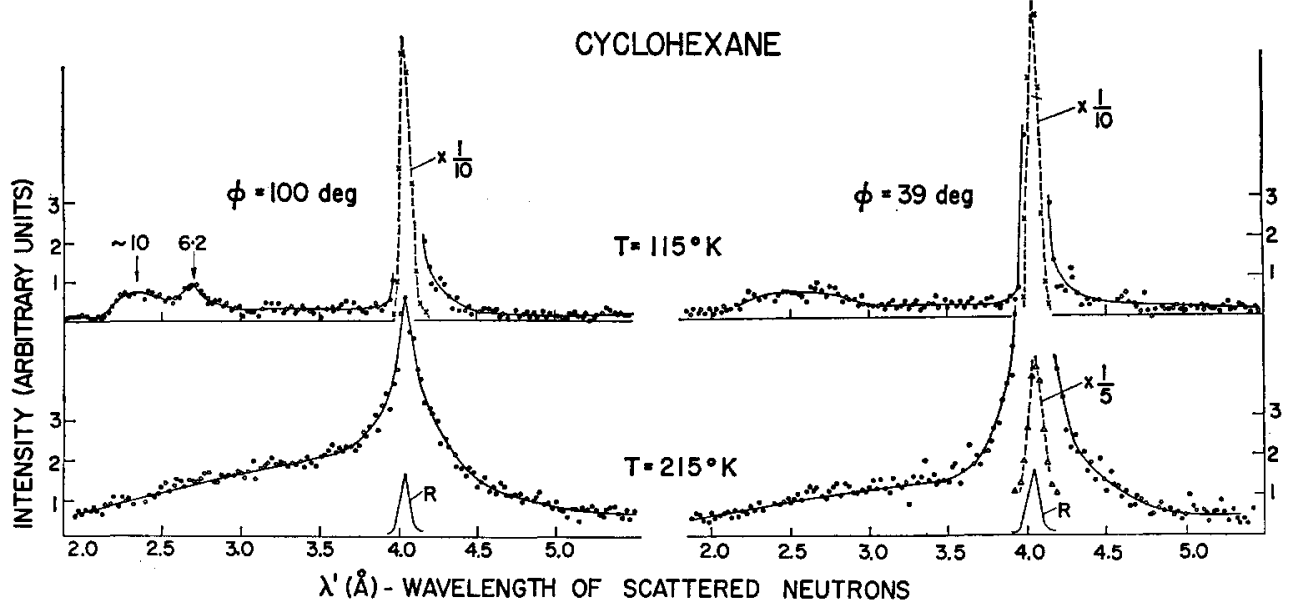

Fig. 1. Plot of intensity vs scattered-neutron wavelength of cyclohexane powder at $115^{\circ}$ and $215^{\circ} \mathrm{K}$, and scattering angles of $39^{\circ}$ and $100^{\circ}$. The position of the peaks are given in $\mathrm{meV}$.

east $10^{5} \mathrm{cps}$ above $186^{\circ} \mathrm{K} .^{9}$ Also 2-2-dimethylbutane has fcc crystal structure in the high-temperature phase $^{10}$ and has a first-order transition at $127^{\circ} \mathrm{K}$. Its behavior as studied by NMR is similar to that of cyclohexane and can also be explained by the existence of almost isotropic reorientation above $127^{\circ} \mathrm{K}$. DABCO undergoes a first-order solid-state transition at $80^{\circ} \mathrm{C}$, as determined by calorimetric measurements. ${ }^{11,12}$ To our knowledge no NMR work has been done on $\mathrm{DABCO}$, and very little $\mathrm{x}$-ray work has been done. ${ }^{13}$

The neutron inelastic-scattering experiments were done with the Chalk River rotating crystal spectrometer. ${ }^{6}$ Neutrons of incident wavelength $4.059 \mathrm{~A}$ were scattered from very thin specimens ( $0.5 \mathrm{~mm}$ thickness) at scattering angles $\phi$ of $19^{\circ}, 39^{\circ}$, and $100^{\circ}$ (corresponding to $Q$ 's of $0.518,1.050$, and $2.400 \AA^{-1}$, respec-

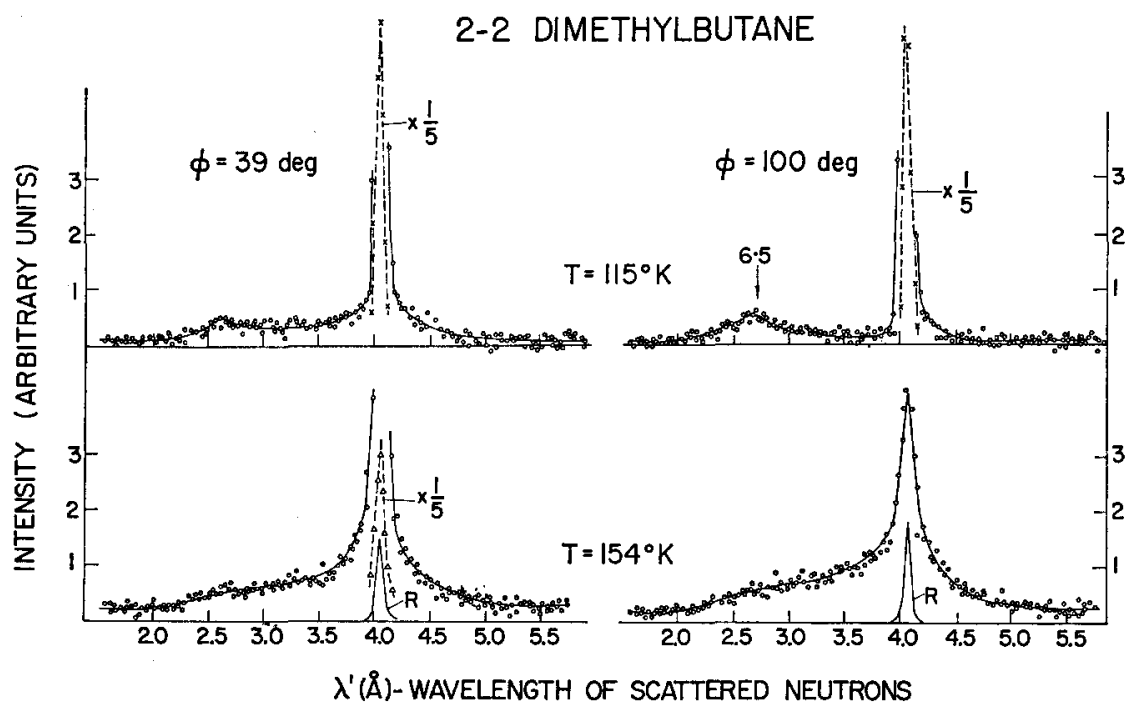

E. R. Andrew and R. G. Eades, Proc. Roy. Soc. (London) A216, 398 (1953)

${ }^{10}$ B. Post, R. S. Schwartz, and I. Fankuchen, J. Am. Chem. Soc. 73, 5113 (1951).

${ }^{11}$ S. S. Chang and E. F. Westrum, Jr., J. Phys. Chem. 64, 1551 (1960).

${ }_{12}$ E. F. Westrum, Phys. and Chem. Solids 18, 83 (1961).

${ }^{13} \mathrm{~S}$. Saki (unpublished work); see reference 12.
FIG. 2. Plot of intensity vs scattered-neutron wavelength of 2-2dimethylbutane at $115^{\circ}$ and $154^{\circ} \mathrm{K}$, and scattering angles $39^{\circ}$ and $100^{\circ}$ The position of the peak is given in meV. 
tively) for liquid cyclohexane and liquid 2-2-dimethylbutane, and at angles of $39^{\circ}$ and $100^{\circ}$ for the solids above and below the transition points. Studies of solid DABCO were made only at $\phi=100^{\circ}$, above and below the transition point. The times of flight of the scattered neutrons with energies near the incident energy were measured in a 178-channel time sorter. For each specimen the net counting rate $N_{0}$ was normalized utilizing:

$$
N_{0}=\left[\left(N-N_{c d}\right)_{s} / M_{s}\right]-T\left[\left(N-N_{c d}\right)_{b} / M_{b}\right]
$$

where $T$ is the transmission of the specimen, $N_{s}$ and $M_{s}$ the monitor counts for measurements with and without specimen, referred to by the subscripts $s$ and $b$, respectively, and $N_{c d}$ refers to measurements made with cadmium in the beam. The transmission in the

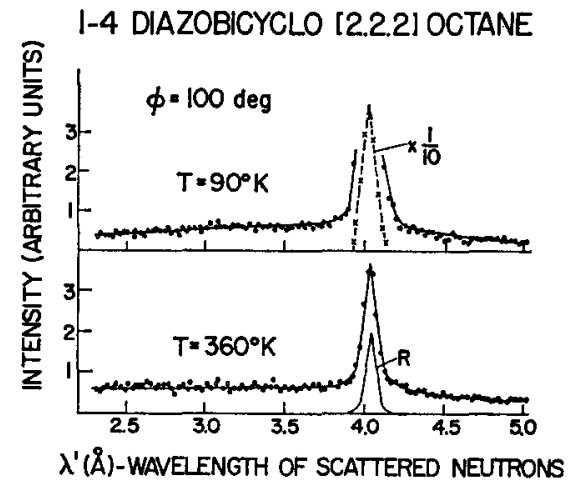

FIG. 3. Plot of intensity vs scattered-neutron wavelength of 1-4-diazobicylco-(2.2.2)-octane at $90^{\circ}$ and $360^{\circ} \mathrm{K}$, and scattering angle of $100^{\circ}$.

forward direction, at $300^{\circ} \mathrm{K}$, was measured before and after the whole series of patterns for each substance was taken, the values obtained are: $T$ (cyclohexane) $=$ $78 \% ; T$ (2-2-dimethylbutane $)=88 \%$ and $T(\mathrm{DABCO})$ $=75 \%$. The normalized counting rates, after correction for counter efficiency, are shown in Figs. 1-5. The patterns for each substance are normalized together, so intensities can be directly compared for each one of them. These figures also show the resolution function $(R)$ for each $Q$, obtained from a series of measured distributions of the elastic incoherent scattering by vanadium metal.

The elastic peaks of solid cyclohexane and 2-2-dimethylbutane show appreciable broadening in passing from the low-temperature to the high-temperature phase. This can be seen very clearly in Figs. 1-2, the difference at the low-temperature being quite drastic. Also, the reduction in width with decreasing $Q$ of the elastic peak of these substances is very marked in the high-temperature phase. This behavior of the diffrac-

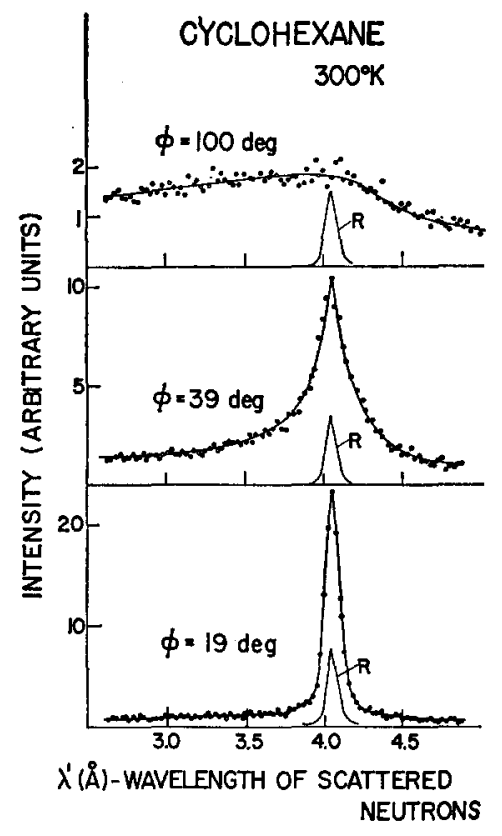

FIG. 4. Plot of intensity vs scattered-neutron wavelength of liquid cyclohexane at $300^{\circ} \mathrm{K}$, and scattering angles of $19^{\circ}, 39^{\circ}$ and $100^{\circ}$.

tion pattern can be attributed to the existence of "quasi-free-rotation." The broad inelastic peaks observed in the low-temperature phase, corresponding to energy transfer $(\hbar \omega)$ of $6.5 \mathrm{meV}$ in 2-2-dimethylbutane and to 6.2 and $10 \mathrm{meV}$ in cyclohexane are probably due to modes of intermolecular vibration. Their disappearance in the high-temperature phase and the broadening of the elastic peak lead to the conclusion that the high-temperature phases of cyclohexane and

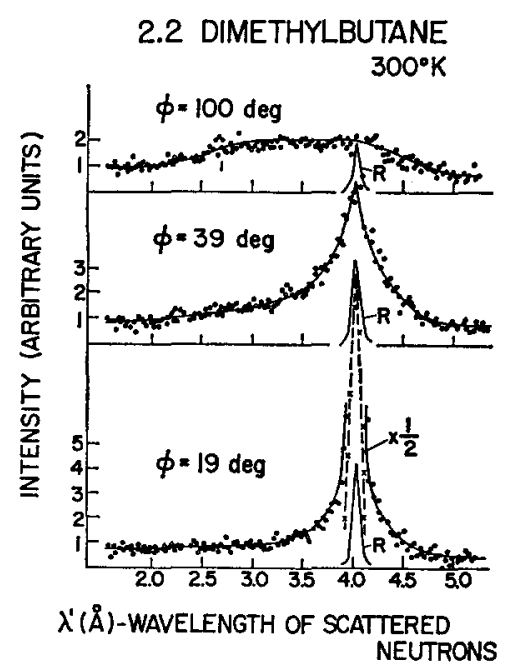

FIG. 5. Plot of intensity vs scattered-neutron wavelength of liquid 2-2-dimethylbutane at $300^{\circ} \mathrm{K}$, and scattering angles of $19^{\circ}, 39^{\circ}$, and $100^{\circ}$. 
2-2-dimethylbutane are dynamically disordered. No evidence of this can be observed in DABCO, the patterns at two temperatures shown in Fig. 3 are of almost identical shape.

Figures 4 and 5 show the diffraction patterns for liquid cyclohexane and liquid 2-2-dimethylbutane at $300^{\circ} \mathrm{K}$. In all patterns the diffusive broadening of the quasi-elastic peak was observed. ${ }^{14}$ Values of the coefficients of self-diffusion $D_{8}$ were calculated on the assumption of a small motions mechanism by which $D_{s}=W / 2 Q^{2}{ }^{14}$ where $W$ is full width at half-maximum in units of $\hbar$ of the quasi-elastic peaks. Assuming that the quasi-elastic peaks are Gaussian (in fact they are approximately Lorentzian), $W$ was measured after correcting for resolution (the shape of the resolution function is approximately Gaussian). The following values of $D_{s}$ were obtained: $D_{s}($ cyclohexane $)=4.5 \times$

${ }^{14}$ B. N. Brockhouse, Suppl. Nuovo Cimento 9, 45 (1958).
$10^{-5} \mathrm{~cm}^{2} / \mathrm{sec}$ and $D_{s}(2-2$-dimethylbutane $)=8.6 \times 10^{-5}$ $\mathrm{cm}^{2} / \mathrm{sec}$, both from the pattern at $\phi=39^{\circ}$. From NMR measurements of spin-lattice relaxation times the following values of $D_{s}$ were obtained: $D_{s}($ cyclohexane $)=$ $1.38 \times 10^{-5} \mathrm{~cm}^{2} / \mathrm{sec}^{15}$ and $D_{s}$ (2-2-dimethylbutane) $=$ $3.41 \times 10^{-5} \mathrm{~cm}^{2} / \mathrm{sec},{ }^{16}$ both at $25^{\circ} \mathrm{C}$. The discrepancy between the $D_{s}$ values from NMR and these obtained here must be mostly due to the effect of the irreversible rotations of the molecules.

\section{ACKNOWLEDGMENTS}

I am indebted to Dr. B. N. Brockhouse for his interest and many helpful suggestions, and to E. Glaser for technical assistance. I would like to thank the National Research Council of Canada for the award of a Fellowship.

${ }^{15}$ D. W. McCall, D. C. Douglass, and E. W. Andrews, J. Chem. Phys. 31, 1555 (1959).

${ }_{16}$ D. C. Douglass and D. W. McCall, Phys. Fluids 2, 87 (1959).

\title{
Revised Tables for Ion-Exchange Kinetics
}

\author{
F. HeLfFERICH \\ Shell Development Company, Emeryville, California
}

(Received 22 October 1962)

\begin{abstract}
Revised numerical solutions of the differential equation for exchange of ions of equal valence and different mobilities are presented for finite slabs and spherical particles under simple initial and boundary conditions. The solutions also apply to sorption and desorption with a particular concentration dependence of the diffusion coefficient. The relations with solutions given by others for semi-infinite media are discussed.
\end{abstract}

$\mathbf{N}$ UMERICAL solutions of the diffusion equation for ion-exchange kinetics have been given previously. ${ }^{1,2}$ These solutions have now been recalculated with a more accurate computer program. The results are given in Tables I and II.

\section{SYMBOLS}

$a=$ constant parameter in Eq. (2)

$C=$ concentration

$C_{\mathrm{A}}=$ concentration of counterion $\mathrm{A}$ in ion exchange

$C_{0}=$ initial or boundary concentration

$D=$ diffusion coefficient, indices $A$ and $B$ refer to individual diffusion coefficients of counterions $A$ and $B$ in ion exchange

${ }^{1}$ F. Helfferich and M. S. Plesset, J. Chem. Phys. 28, 418 (1958).

${ }_{2}^{2}$ F. Helfferich, J. Phys. Chem. 66, 39 (1962).

$$
\begin{aligned}
D_{0} & =\text { diffusion coefficient at zero concentration } \\
k & =\text { constant in } \mathrm{Eq} .(5) \\
r & =\text { radial space coordinate in spheres } \\
r_{0} & =\text { sphere radius } \\
t & =\text { time } \\
x & =\text { space coordinate normal to surface in finite } \\
& \text { slabs } \\
x_{0} & =\text { thickness of finite slab } \\
\tau & =\text { dimensionless time }
\end{aligned}
$$

The tables list solutions of the diffusion equation,

$$
\partial C / \partial t=\operatorname{div}(D \operatorname{grad} C)
$$

with a concentration-dependent diffusion coefficient,

$$
D=D_{0}\left[1 /\left(1+a C / C_{0}\right)\right],
$$

for various values of the parameter $a$ and with the 